\section{Wie den Impfstoff beziehen?}

Im Allgemeinen wird der Grippeimpfstoff über Sprechstundenbedarf bei einer Apotheke der Wahl bezogen. Dazu müssen die länderspezifischen Besonderheiten bei der KV erfragt werden, weil in einigen Bundesländern die Krankenkassenverbände den Grippeimpfstoff ausgeschrieben haben. In diesen Fällen können nur die vertraglich vereinbarten Impfstoffe über den Sprechstundenbedarf bezogen werden. Diese Regelung gilt auf jeden Fall für: KVB, KVBB, KVHH, KVN, KVSH.

Alle anderen Impfstoffe gelten wegen des fehlenden Abschlages als unwirtschaftlich und müssen individuell auf Kassenrezept auf den Namen des Geimpften verordnet werden. In diesen Fällen sollte der Vertragsarzt eine entsprechende Begründung dokumentieren, damit er die Abweichung - z. B. wegen Hühnereiweißallergie - belegen und den Vorwurf der Unwirtschaftlichkeit widerlegen kann.

\section{Dokumentieren, dokumentieren, dokumentieren ...}

Ohnehin ist bei der Impfung auf eine lückenlose Dokumentation zu achten, da die Kassen die Anzahl der bezogenen Impfstoffe in Relation zu den abgerechneten Impfziffern stellen. Gibt es hier eine relevante Differenz - mehr bezogene Impfstoffe als abgerechnete Impfleistungen - steht eine Regressforderung ins Haus.

Eine Zusammenstellung der Krankenkassen, die die Grippeschutzimpfung als Satzungsleistung für alle Versicherten übernehmen, finden Sie unter: http://www.krankenkassen.de/ gesetzliche-krankenkassen/leistungengesetzliche-krankenkassen/praevention-vorsorge-krankenkassen/listegrippeimpfung/

Helmut Walbert .

Wann ist es Zuweisung gegen Entgelt?

\title{
Dämpfer aus Karlsruhe für Teilgemeinschaften
}

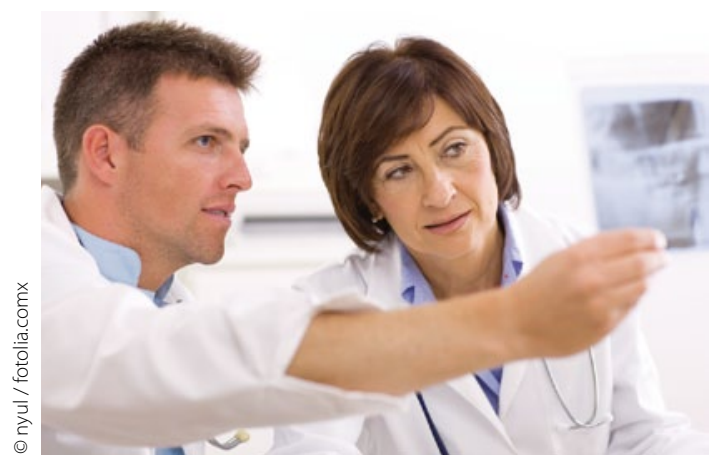

Entscheidend ist, wer alles an einen Facharzt überweist.

Die streitige Gestaltung wäre also zulässig gewesen, sobald die Radiologen ihre Leistungen auch dann auf Rechnung der Teilgemeinschaftspraxis erbracht hätten, wenn der veranlassende Arzt nicht Mitgesellschafter der Teilgemeinschaftspraxis ist.

\section{Wie dürfen Fach- und Hausärzte ihre Kooperation gestalten, ohne dass sie gegen das Verbot der Zuweisung gegen Entgelt verstoßen? Das Ober- landesgericht Karlsruhe zieht eine klare Grenze für Teilberufsausübungs- gemeinschaften.}

- Die Zusammenarbeit im Rahmen einer Teilberufsausübungsgemeinschaft ist Ärzten erlaubt, darf aber nicht einer Umgehung des Verbotes der Zuweisung gegen Entgelt dienen. Dieser Fall liegt z. B. vor, wenn sich der Beitrag eines Arztes in der Kooperation auf das Erbringen medizinisch-technischer Leistungen auf Veranlassung der übrigen Mitglieder einer Teilberufsausübungsgemeinschaft beschränkt, so das Oberlandesgericht (OLG) Karlsruhe. Eine Teilberufsausübungsgemeinschaft aus Nordbaden konnte nicht einwandfrei darlegen, dass die teilnehmenden Radiologen für die Gemeinschaft auch Leistungen erbringen, die nicht von Mitgesellschaftern veranlasst wurden (Az.: 6 U 15/11).

Es kommt also darauf an, ob der Radiologe auch für Ärzte außerhalb der Gemeinschaft Leistungen erbringt und diese dann als Mitglied der Teilgemeinschaftspraxis, also nicht für seine daneben bestehende eigene Praxis abrechnet.

\section{Beispielfall einer Kooperation}

Ein Facharzt und ein Laborarzt könnten sich vollumfänglich zu einer Gemeinschaftspraxis verbinden. Der Facharzt will primär an den Umsätzen "seiner" Laborproben partizipieren und nicht in die Haftung für die teuren Laborgeräte eintreten. Der Laborarzt will eigentlich nur den Facharzt als Einsender an sich binden, aber nicht seine gesamten Laborgewinne mit ihm teilen.

Weil sich bei dieser Interessenlage das Gemeinschaftliche letztlich auf wirtschaftliche Überlegungen im Bereich der überweisungsgebundenen, also in gewisser Weise steuerbaren Leistungen reduziert, beurteilen die Berufsordnungen die Zulässigkeit anders als wenn sich zwei Ärzte vollumfänglich zur gemeinsamen Berufsausübung zusammenschließen.

Umgekehrt ist es aber sowohl freiberuflichen Ärzten als auch in MVZ angestellten Ärzten untersagt, an einer Teilberufsausübungsgemeinschaft mitzuwirken, die sich bei medizinisch-technischen Leistungen darauf beschränkt, diese nur auf Veranlassung anderer Mitgesellschafter, und eben nicht auf Überweisung aller Ärzte, zu erbringen.

INGO PFLUGMACHER . 